\title{
Analytical Calculation and Analysis of Main Generator Inductance of Aero Brushless Three-stage Synchronous Starter/Generator System
}

\author{
Jixiang Wang , Weiguo Liu and Zan Zhang \\ School of Automation, Northwestern Polytechnical University,710072, Xi'an, China.
}

\begin{abstract}
Precise electro-magnetic parameters are significant in the investigation of characteristic for brushless synchronous starter/generator system, especially the inductance during the starting process.This paper presented an analytical method for inductance calculation, which analysed the influence of stator windings' parallel structure on inductance calculation and took the skewed slot structure into consideration. Due to the skewed slot structure, applicable two-dimensional coordinate systems were established.This proposed method could save time in calculation according to the simplification to some extent. The results of analytical method were presented and compared with the simulation results. The reason for the differences has been analyzed.
\end{abstract}

\section{Introduction}

Nowadays, more and more attention has been paid to the integrated starter/generator system which is significant to the aircraft power supply system. The three-stage starter/generator system generally consists of a pilot exciter, a main exciter and a main generator.

The main generator adopts the skewed slot structure which can improve the performance of the system by reducing the harmonics and the torque ripple. In order to analysis the motor with skewed slot structure, finite element method (FEM) or analytical calculation method are adopted. In FEM, a three-dimensional model or equivalent multiple two-dimensional models should be established, which makes the simulations complex and time-consuming ${ }^{[1-5]}$. In this paper, a two-dimensional coordinate system is proposed based on magnetic circuit method, and then on the basis of the motor structure all work was investigated in this coordinate system to calculate the parameters.

\section{The influence of motor structure on inductance calculation}

Considering the main generator adopts the parallel branches for stator wingdings, some simplification is made to analyse the influence. It's assumed that concentrated windings are employed and saturation is ignored. Meanwhile the MMF drop on the core of stator and rotor in the loop and the leakage flux are ignored.

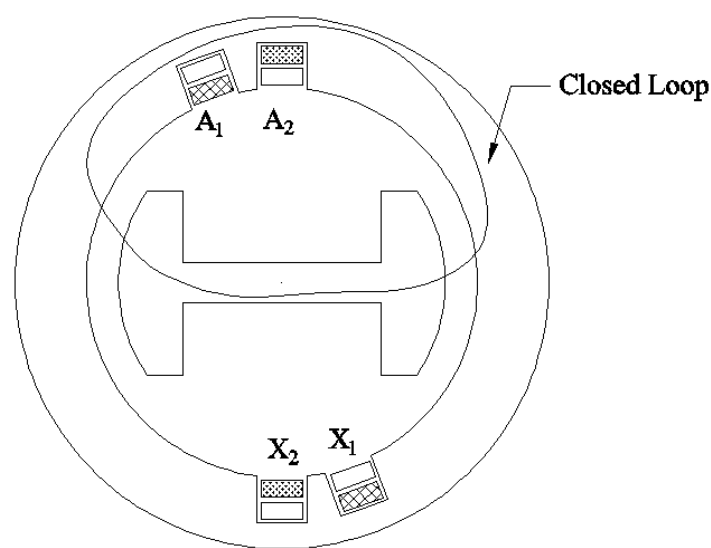

Figure 1. Simplified diagram of concentrated windings with parallel branches

As shown in Fig 1, current $i$ is given to each parallel branch of phase A and then to phase A. On the basis of Ampere circuital theorem, the magnetic motive force (MMF) under these modes can be expressed as:

$$
\left\{\begin{array}{l}
F_{A X 1}=N_{1} i \\
F_{A X 2}=N_{2} i \\
F_{A}=\left(N_{1}+N_{2}\right) i / 2=N i
\end{array}\right.
$$

Due to same number of turns for each parallel branch, $N_{1}=N_{2}=N$, so that $F_{\mathrm{AX} 1}=F_{\mathrm{AX} 2}=F_{\mathrm{A}}$. Meanwhile, the MMF can be written as $F=2 H l$, where $H$ is the airgap magnetic field intensity and $l$ is the length of the airgap. Due to the assumption, so $H_{\mathrm{AX} 1}=H_{\mathrm{AX} 2}=H_{\mathrm{A}}$ which means $B_{\mathrm{AX} 1}=B_{\mathrm{AX} 2}=B_{\mathrm{A}}$. The flux is equal because of the same cross-sectional area, which means $\Phi_{A X 1}=\Phi_{A X 2}=\Phi_{A}$. According to the magnetic circuit ohm's law, 
$R_{\mathrm{AX} 1}=R_{\mathrm{AX} 2}=R_{\mathrm{A}}$ where $R$ is the magnetic resistance. The inductance should be described as:

$$
\left\{\begin{array}{l}
L_{A X 1}=\frac{\varphi_{A 1}}{i}=\frac{N_{1} \Phi_{A X 1}}{i}=\frac{N_{1} F_{A X 1}}{i R_{A X 1}}=\frac{N_{1} N_{1} i}{i R_{A X 1}}=\frac{N_{1}^{2}}{R_{A X 1}} \\
L_{A X 2}=\frac{\varphi_{A 2}}{i}=\frac{N_{2} \Phi_{A X 2}}{i}=\frac{N_{2} F_{A X 2}}{i R_{A X 2}}=\frac{N_{2} N_{2} i}{i R_{A X 2}}=\frac{N_{2}^{2}}{R_{A X 2}}(2) \\
L_{A}=\frac{\varphi_{A}}{i}=\frac{N \Phi_{A}}{i}=\frac{N F_{A}}{i R_{A}}=\frac{N N i}{i R_{A}}=\frac{N^{2}}{R_{A}}
\end{array}\right.
$$

Finally, $L_{\mathrm{AX} 1}=L_{\mathrm{AX} 2}=L_{\mathrm{A}}$ is derived from the magnetic circuit analysis above. It can be shown that the self-inductance of the phase A winding is equal to that of each parallel branch on the assumptions above which can simplify the calculation. However motor usually adopts distributed windings and the saturation can't be ignored, so they are not equal actually.

Damper wingdings are installed in the rotor of main generator to weaken the harmonics during the starting process. Since the damper windings can't be open, the inductance of armature windings can't be measured directly. It means that the measurement data needs further analysis to get the actual value ${ }^{[6]}$.

\section{Analytical calculation method for inductance of windings}

\subsection{Coordinate Systems with Skewed Slot Structure}

In order to describe the MMF and permeance, appropriate coordinate systems must be established. It's assumed that the stator slots are skewed by $\alpha_{c}\left(\alpha_{c} \neq 0\right)$ in mechanical radian, the axial length of motor core is $d$ and the length of the corresponding bevel edge is $h$.

The coordinate system of stator is a two-dimensional oblique coordinate system. Its abscissa axis extends along the stator circle and vertical axis extends along the skewed slots, whose origin is set by the intersection of one stator slot's symmetrical line and the abscissa axis. The coordinate system of rotor is a two-dimensional rectangular coordinate system. Its abscissa axis extends along the rotor circle and vertical axis extends along the $\mathrm{d}$-axis of rotor, whose origin is set by the intersection of the $\mathrm{d}$-axis and the abscissa axis, as shown in Fig $2^{[7]}$.

d-axis

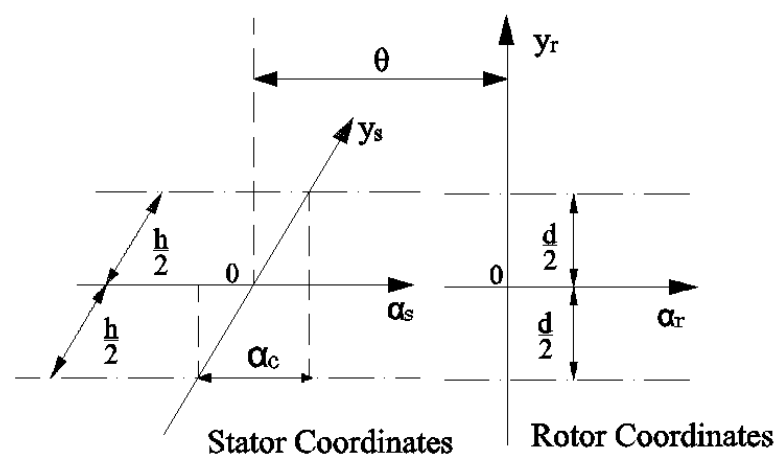

Figure 2. Coordinate systems with skewed slot structure
It is assumed that the rotor coordinates run ahead of the stator coordinates by the mechanical radian $\theta$, as shown in Fig 2. Therefore, the conversion from one coordinate system to another can derive itself from the systems :

$$
\left\{\begin{array}{l}
\alpha_{s}=\theta+\alpha_{r}-\frac{\alpha_{c}}{d} y_{r} \\
y_{s}=\frac{h}{d} y_{r}
\end{array}\right.
$$

\subsection{Airgap magnetic permeance}

Considering the effort of the stator slot opening only, the permeance is given as follow:

$$
\lambda_{s}\left(\alpha_{s}, y_{s}\right)=\frac{\mu_{0}}{\delta} \sum_{v=0}^{+\infty} a_{s v} \cos \left(v Z \alpha_{s}\right)
$$

Where $\mu_{0}$ is the magnetic permeance in vacuum, $\delta$ is the mean length of airgap, $Z$ is the number of stator slots, $a_{s v}$ is the magnitude of $v$-order harmonic of airgap magnetic permeance considering the effort of the stator slot opening only ${ }^{[8]}$.

Considering the rotor saliency and rotational symmetry, the permeance is similarly given as follow:

$$
\lambda_{r}\left(\alpha_{r}, y_{r}\right)=\frac{\mu_{0}}{\delta} \sum_{k=0}^{+\infty} b_{r k} \cos \left(2 p k \alpha_{r}\right)
$$

Where $p$ is the number of pole-pairs, $b_{r k}$ is the airgap magnetic permeance's magnitude of $k$-order harmonic only considering rotor saliency. The rotational symmetry determines that the permeance should be expressed as an even function.

Finally the permeance can be calculated in the superposition method:

$$
\lambda=\lambda_{s} \lambda_{r}=\frac{\mu_{0}}{\delta} \sum_{v=0}^{+\infty} a_{s v} \cos \left(v Z \alpha_{s}\right) \sum_{k=0}^{+\infty} b_{r k} \cos \left(2 p k \alpha_{r}\right) \text { (6) }
$$

\subsection{Magnetic motive force}

The fundamental harmonic of MMF for single phase of stator windings in the stator coordinate system can be expressed as follow ${ }^{[9]}$ :

$$
F_{s i}\left(\alpha_{s}, y_{s}\right)=I_{s i} F_{s 1} \cos \left(p \alpha_{s}+p \alpha_{s i}\right), \quad i \in\{a, b, c\} \text { (7) }
$$

\subsection{Airgap flux linkage and inductance}

According to (6) and (7), the airgap flux density inspired by single phase of stator windings with coordinate transformation is given as follow:

$$
\begin{aligned}
B_{s} & \left(\alpha_{s}, y_{s}\right)=\lambda F_{s i}\left(\alpha_{s}, y_{s}\right) \\
= & \frac{\mu_{0}}{\delta} F_{s i}\left(\alpha_{s}, y_{s}\right)\left\{\sum_{v=0}^{+\infty} a_{s v} \cos \left(v Z \alpha_{s}\right) \sum_{k=0}^{+\infty} b_{r k} \cos \left[2 p k\left(\alpha_{s}-\theta+\frac{\alpha_{c}}{h} y_{s}\right)\right]\right\}
\end{aligned}
$$

The flux linkage of one stator winding can be expressed as follow: 


$$
\psi_{s}\left(\alpha_{s c 1}, \alpha_{s c 2}\right)=\frac{d R_{\delta} N_{s c}}{h} \int_{\alpha_{s c 2}}^{\alpha_{s c 1}} \int_{-h / 2}^{h / 2} B_{s}\left(\alpha_{s}, y_{s}\right) d y_{s} d \alpha_{s}
$$

Where $R_{\delta}$ is mean radius length of airgap, $N_{\mathrm{sc}}$ is the turn number of the stator winding, $\alpha_{s c 1}$ is the upper side's coordinate value of winding, $\alpha_{s c 2}$ is the coordinate value of lower side.

After the accumulation of flux linkage through one winding, the inductance should derive itself from the definition :

$$
L_{s}=\sum \psi_{s} / I_{s i}
$$

The same procedure can be easily adapted to obtain rotor inductance parameters.

\section{Verification Results}

\subsection{Results of Self-inductance}

First, the analytical method is employed on a salient-pole synchronous motor, and then FEM simulations without damper windings are carried out. The results are shown in Fig 3.

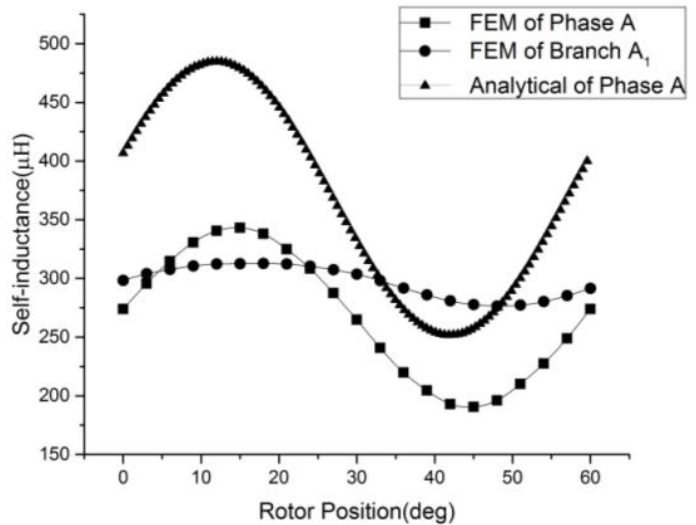

Figure 3. Self-inductance of phase A armature winding

\subsection{Results of mutual-inductance}

The results of mutual-inductance obtained by these two methods are shown in Fig 4.

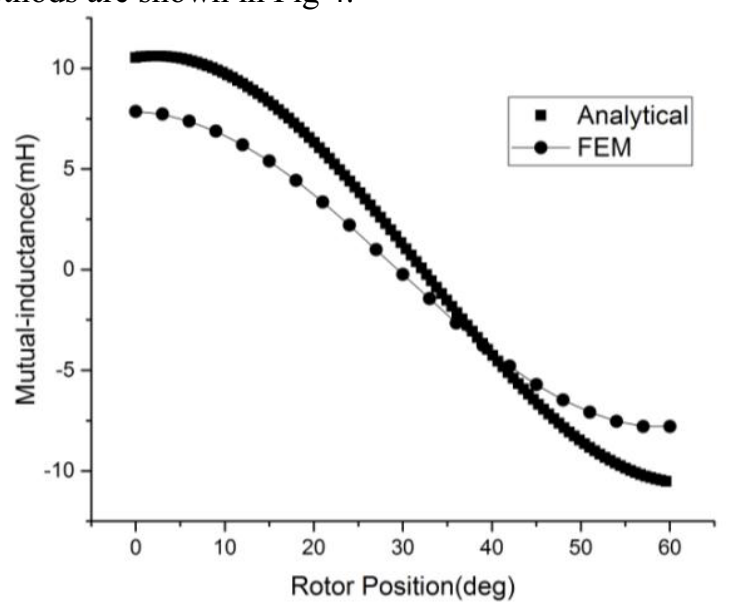

Figure 4. Mutual-inductance between phase A and field windings

\subsection{FEM Simulation Results and Measured Data with Damper Windings}

The self-inductance parameters are measured. Meanwhile, the FEM simulations of the established model with damper windings are carried out. The results are shown in Fig 5.

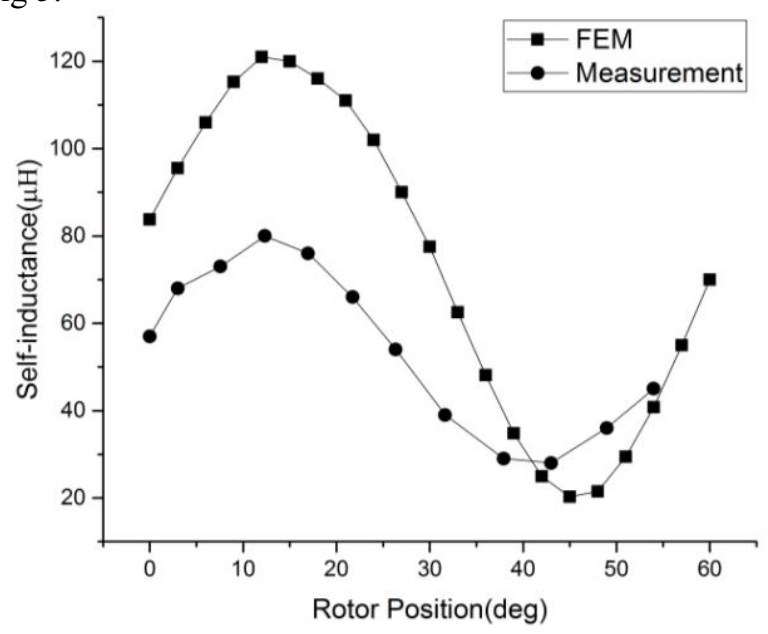

Fig 5. Self-inductance of FEM simulation and measurement

\subsection{Analysis and Follow-up Work}

First, the length of airgap under poles was employed in the analytical calculation instead of the mean length, so the results of analytical method are 1.36 times larger than FEM simulation results. Then, the FEM simulation results of self-inductance for phase A and branch $\mathrm{A}_{1}$ can't match well because of different saturation resulted by different loads actually. Due to the quick and time-saving calculation, only the fundamental harmonic of MMF is employed. Considering the damper windings, the reason for the differences between the inductance parameters of FEM simulation and measurement is that the simulation models were established without skewed slots and the so-called measured self-inductance parameters are not equal to actual parameters.

In the analytical calculation, accurate parameters should be employed. At the same time, one method should be adopted to analyze the measurement data and identify the actual value of inductance.

\section{Conclusion}

A fast analytical calculation method of inductance based on magnetic circuit method was presented in this paper. Based on the analysis of MMF and permeance under the established two-dimensional coordinate systems, the inductance of motor with skewed slots was calculated and compared with the FEM simulation results. To some extent, the measurement data can verify the validity of FEM simulation models and the analytical method. Then more parameters of motor can be calculated such as the electromagnetic torque and analyzed next. This method can save time effectively and be applicable to other kinds of motor based on the established two-dimensional coordinate systems. 


\section{References}

1. Shima K, Ide K, Takahashi M., Finite-element Calculation of Leakage Inductances of a Saturated Salient-pole Synchronous Machine with Damper Circuits[J].IEEE Transactions on Energy Conversion,2002,17 (4) :463-470.

2. Chen Y. S., Zhu Z. Q., Howe D., Calculation of d- and q-Axis Inductances of PM Brushless ac Machines Accounting for Skew[J]. IEEE Transactions on Magnetics, 2005,41 (10):3940- 3942.

3. Williamson Stephen, Flack T.J., Volschenk A.F.. Representation of Skew in Time-stepped Two-dimensional Finite-element Models of Electrical Machines[J].IEEE Transactions on Industry Applications, 1995,31(5):1009-1015.

4. Dziwniel P., Piriou F., Ducreux J.P., et al, A Time-stepped 2D-3D Finite Element Method for Induction Motors with Skewed Slots Modeling[J]. IEEE Transactions on Magnetics, 1999,35(3):1262-1265.
5. Akbari H.R., Sadeghi S.. Calculation of Inductances of Induction Machines Under Axial Nonuniformity Conditions[C]. IEEE International Symposium on Industrial Electronics, Vigo :IEEE,2007.1113-1118.

6. Qiao Mingzhong, Zhang Xiaofeng, Ren Xiuming, Measurement and Calculation Winding Parameters for Multi-Phase High-Power Propulsive PMSM with Damping Windings [J].Transactions of CHINA Electrotechnical Society,2003,18(6):37-41.

7. Ou Yangbin, Liu Dezhi, Zhai XIaofei, Analytical Calculation of Inductances of Windings in Electrical Machines with Slot Skew [C]. XIX International Conference on Electrical Machines - ICEM 2010, Rome.

8. Gao Jingde, Wang Xiangheng, Li Fahai, Analysis of AC Machines and Systems, 2nd ed., Beijing: Tsinghua University Press, 2005, pp. 458-462.

9. Gu Chenglin, Chen Qiaofu, Xiong Yongqian, Electrical Machinery, 3nd ed., Wuhan: Huazhong University of Science and Technology Press, 2010, pp. 392-397. 